\title{
Structural Dilemma in Health System Reform in the Chinese Mainland and Emerging of Structure-Based Bioethics Paradigm
}

\author{
Liu Jitong, Zhang Dongqi \\ Department of Health Policy and Management, School of Public Health, Peking University, Beijing, China \\ Email: zhangdongqi@bjmu.edu.cn \\ Received April $7^{\text {th }}$, 2013; revised May 23 $3^{\text {rd }}, 2013$; accepted June $13^{\text {th }}, 2013$
}

Copyright (C) 2013 Liu Jitong, Zhang Dongqi. This is an open access article distributed under the Creative Commons Attribution License, which permits unrestricted use, distribution, and reproduction in any medium, provided the original work is properly cited.

\begin{abstract}
After reviewing the historical development of ethics and individual-based bioethics in Western countries, and comparing that in China, this paper starts from the extreme Beijing Tongren Case and the dilemma in the reform of health system in the Chinese mainland, and puts forward the topics of bioethics and medical professionalism under the circumstances of globalization, global health, and global health system reform. It compares the individual-based bioethics in Western countries and the structure-based bioethics in the Chinese mainland, and discusses the lessons of structure-based bioethics for other countries. The historical experience of health system reform in the Chinese mainland indicates that the health care system is welfare in nature, which is crucial; that the welfare health care system is the systematic foundation of medical professionalism; that there should not be direct economic interest generated in doctor-patient relationship since the marketization of health financing may lead to not only the structure intension of doctor-patient relationship, but also a total collapse of the medical professionalism. Therefore the Health Finance rather than Health Economics should be the material foundation of medical professionalism. The paper also points out that the main determinants of medical professionalism are not individual medical professionals, but the outcome of interaction between socio-cultural institutions and individual professionals, in which the sociality and structure are the most important components.
\end{abstract}

Keywords: Health System Reform in the Chinese Mainland; Bioethics; Individual-Based Bioethics; Structure-Based Bioethics; Medical Professionalism

\section{Extreme Case in Tongren Hospital and the Structural Dilemma in Health System Reform in the Chinese Mainland}

Beijing Tongren Hospital 同仁医院, the best known eye hospital in the Chinese mainland, witnessed an intensified doctor-patient relationship in September, 2011, when a chief physician was hacked more than a dozen times by one of her patients (China Youth Daily, 2011). This tragedy aroused heated discussion throughout the country, particularly loud cries from doctors for the safeguard of their life during work. It is not an accident but a typical case that reflects a serious social problem. Such seriously disordered work environment is related to the bioethical dilemma in health system reform on a systematic and structural level.

There are no regular statistical data in this regard in the Chinese mainland, but according to some statistics in 2002 and 2003 from medical agencies at all levels of China Hospital Management Association 中国医院管理协会, medical malpractices occurred in $98.43 \%$ hospitals, and the case number in each hospital kept increasing. When dealing with the medical malpractices, $73.5 \%$ patients or their families intensified the conflicts rather than resorted to the law. In addition, hospital violators ("Yinao 医闹” in Chinese) have become a new group of people who take benefit from the help they offer to patients to initiate legal proceedings or to take radical actions to violate hospital regulations. Nearly half of the hospitals were vandalized by patients or their families, which resulted in 34.64\% cases of injury of medical workers. In Guangdong Province, more than half medical workers experienced violence twice or more in one year. From $86 \%$ to $96 \%$ hospitals claimed cases where patients complained about the hospital, refused to discharge from hospital or pay medical expenses. As for the amount of compensation claimed by patients, it is positively related to the levels of hospital. For example, $5 \%$ of the threelevel hospitals bear $¥ 500,000$ to $1,000,000$ compensation while the percentage for the two-level and one-level hospitals are $1.1 \%$ and $0 \%$ respectively; $75 \%$ of the three-level hospitals offer less than $¥ 100,000$, while the compensation from $92.5 \%$ of the two-level hospitals fall in this range (China Hospital Management Association, 2003).

The reform and opening up policy since the late 1970s has promoted the Chinese mainland to the second largest economic entity around the world, but the development has also brought problems and resultant disputes over politics, laws, economics, society, culture and ethics. The society has become high-risk and filled with social contradictions. The medical field has become one of the industries with most serious problems. The medical and philosophical foundation, medical professionalism and bioethics are in structural dilemma, becoming the most urgent social topics (Xu Zhiwei, 2006). At a time when nationwide efforts are being made to "establish a harmonious society 
focusing on improving people's livelihood”, and, especially, in the social context where physical and mental health needs have become the most important social needs (Liu Jitong, 2004a), people expect more from doctors, which is widening the gap between doctors' behavior and the social expectation. More specifically, because of the "poor access and high fee", structural tension in doctor-patient relationship has intensified. Occurences of hurting, abusing, and even killing doctors further complicate the structural dilemma of bioethics (Sun Shuyin, 2011).

Why does the health system reform in the Chinese mainland lead to the structural tension in doctor-patient relationship? What is the reason behind the hurting, abusing and killing doctors in the Chinese mainland? Is it due to the decreasing professionalism of Chinese doctors or the common challenge faced by doctors around the world? How to build harmonious doctorpatient relationship, and re-establish the medical professionalism? If individual-based professional ethics does not adequately explain such dilemma in the health system reform, what is to be blamed and how to solve the problem? And what lessons can other countries and global medical community draw from this? The above questions are to be discussed in this paper.

\section{Western Culture, Tradition and the Paradigm of Individual-Based Bioethics}

Reform in health system prevails in the world. Many countries are looking for new ways to maximize the benefits for the patients and take care of the doctors' rights at the same time. However, the situation in the Chinese mainland shows insufficient protection for doctors. Western countries focus more on individualism, which is also manifested in medical field. Their practices cannot be cited to explain, still less to solve, the problems in the Chinese mainland, a region where different even opposite situations exist at the same time; therefore a structural change should be done.

The historical development of Western ethical mode includes natural ethics, sanctification discipline, individualism, diversification, and so on, which clearly describes the transitional locus from virtue of the rulers to the individual-based bioethics, with individuals eventually being the leading subject in both modes (Macintyre, 1967; Engelhardt, 1996). The paradigm of individual-based bioethics just reflects the structural features in Western society, and results from the interaction among society, politics, economics, culture and individual in the West, which could hardly serve as an explanation of the situations in China.

The long history of traditional virtues and moral philosophy in pre-modern China cultivated multiple ethical modes, like Confucian ethics in Spring and Autumn 春秋 Period (770-476 BC), Buddhist Ethics as of the Eastern Han 东汉 Dynasty (25-220 AD), Daoist 道 Ethics after the Three Kingdom 三国 Period (220-280 AD), social ethics focusing on Christian Ethics after the first Opium War in 1840, professional ethics focusing on business ethics, and revolutionary ethics centering on Marxism and socialism in modern era. Generally speaking, different moral philosophies and codes of conduct share the common ground of "group ethics" except for the pursuit of self development and self perfection. Therefore there exist wide differences and sharp contrasts between China's ethics and the individual-based bioethics in Western society.

\section{Structure-Based Bioethics and Its Differences with Individual-Based Bioethics}

Structuralism, as one of the important social theories in 20th century, relates with multiple subjects including sociology, linguistics, anthropology, developmental psychology and biology. Structure-based bioethics tries to explain people's moral ideas and codes of conduct from the perspective of social structure. It is, rather than a brand new theoretical innovation, an inevitable product of the bioethical dilemma of the health system reform in the Chinese mainland.

The word "structure" means diversification, systematicness, comprehensiveness, and institutionalization. It can be observed in many circumstance, contents, approaches, and cause-effect relations in human life.

Generally speaking, there are six major components in the system of structure-based bioethics, reflecting its structural and systematic feature from different aspects.

First of all, the orientations of moral philosophy of structurebased bioethics: by that we refer to people's moral ideas that result from the integration of objective reality and subjective reality. Secondly, the structure-based bioethics is characterized by its realism, collectivism, high integration of human nature and institutional construction. Thirdly, the research objective of structure-based bioethics is not just to identify what people should do, but more importantly, to understand the social reality that functions as a context for people's moral ideas and codes of ethics. This is the foundation to understand the codes of conduct (Sidgwick, 1901). Fourthly, the research scope of structure-based bioethics is rather extensive. It includes not only macro social environment and history, cultural tradition and moral philosophy, but the social environment, institutional arrangement and policy framework, and service system for personal development, particularly the interaction between the individual and the social environment (Smart \& Bernard, 1983). In other words, the structure-based bioethics explores the moral philosophy and code of ethics from the perspective of macro society and social structure. Fifthly, the research perspectives of structure-based bioethics are not limited to philosophical analysis. It features an interdisciplinary perspective from which the universal law of the development of moral philosophy will be examined. Sixthly, the major function of structure-based bioethics is oriented more towards an objective to change the world rather than one to explain it. So it is meant to contribute to establishing a harmonious society and harmonious interpersonal relationship, doctor-patient relationship in particular, through system building (Zhao, 2005).

The individual-based bioethics is at once a direct and indirect antithesis to the structure-based bioethics. The systematic and structural features of structure-based bioethics-as distinct from individual-based bioethics - are the focus of this paper. The structure-based bioethics has a wide research scope that covers people's social life and individual code of ethics, especially the interaction between code of ethics and individual behavioral mode at specific time and under specific circumstances. It aims to explore the social determinants that influence people's moral philosophy, moral ideas and code of ethics, and to develop a new system of moral philosophy theory ( $\mathrm{Li} \mathrm{Qi}$ 1994). The following aspects show the differences between the two based on an "ideal model", which may ignore artificial exaggeration or minimization.

First of all, the most fundamental and prominent characteristic of structure-based bioethics is "sociality or socialization", but not "individuality or individualization". The basic unit of the subject engaged in moral activities is socialized individual. In a socialized individual or one person's morality, one may 
catch a glimpse of the whole society or the overall social situation, because individual moral ideas and code of ethics is capable of reflecting the general social situation at certain circumstances. The "individual morality" is "social morality" in essence, so it is not something "personal". For instance, hurting or abusing doctors is not an isolated act of any individual. Rather it is a socialized phenomenon generated and caught in the web of social meanings and social relations. Therefore more focus should be put on the social meanings behind individual code of ethics and behavioral pattern, and the social environment one is in.

Secondly, the most fundamental and prominent characteristic of structure-based bioethics is "social structure", but not purely individualized life. It is widely known that moral ideas, code of ethics and moral judgment are fundamentally formulated, enacted, or delivered on individual basis, but this does not mean individuals are merely independent units in this regard. It should be noted that individual behaviors are not pure personal. On the contrary, they are behaviors of social actors of macro situation and micro relations. And in that capacity, they are the typical manifestations of structuralized interaction of society. A perspective of social structure emphasizes the interrelation across the systems and the parts therein. For example, the distrust between patients and doctors has become a patterned thinking in Chinese society, which is the result of structuralized interaction of society and accumulation of social influences.

Thirdly, two other important characteristics of structure-based bioethics are its diversity of origin and coexistence of various moral believes and practices. For instance, the traditions of ethics in contemporary China are manifold, including ancient Chinese moral philosophy, modern western Christian ethics, modern business ethics of capitalism, Marxism and revolutionary ethics, socialist ethics from former Soviet Union, modern social and moral philosophy, post-modern social and ethics, and other ethics from other nations and countries (Sun Muyi, 2007). However the historical development of Western social moral philosophy and individual-based bioethics indicates a singular line of individual moral philosophy in Western countries.

Fourthly, another important characteristic of structure-based bioethics is collectivism, which consists of different smaller groups and occupations, illustrating the socialized, structured, and diversified social interaction. After China's reform and opening up policy, the social situation has turned from highly unification to diversity. Larger gaps come into being among residents from different geographic locations, different organizations, and different occupations, which result in social inequity and social injustice (Liu Jitong, 2004b). More importantly, compared with the classical individualism in Western countries and the collectivism before the reform and opening up in the Chinese mainland, the current Chinese society is producing a very unique "structural individualism" during its social structural transform. By that we mean the organization in which one works, his/her position, and the resources and power relations that decide his/her living condition. In the medical field, this perception induces people to take a part for the whole. They may think that all doctors are the same as some particular ones who exhibit insufficient medical professionalism and relatively low morality. This is definitely unfair to the rest health workers.

The last characteristics of structure-based bioethics are its historicity and cultural relativity. The former indicates the periodical features of a specific historical development, and the latter reflects social relativity of moral philosophy and bioethical practices. The Chinese mainland is undergoing a transition from a traditional to a modern society, which defines the current phase of development. The moral philosophy and bioethics are also in this transitional process. For example, the collectivism and moral philosophy in the Chinese mainland is passing through structural individualism, and heading to the modern individualism typical in the West. But Chinese moral philosophy and bioethical thoughts are significantly different from their Western counterparts. They are mainly characterized by situational and traditional implications, which uphold social harmony, doctrine of the mean, the unity of heaven and humanity, and diversity-orderly structure. All of these function to help those in distress and aid those in peril and so on (Fletcher, 1966). Generally speaking, the historical development and cultural characteristics promote the moral philosophy and bioethics into structure-based bioethics.

The following Table 1 shows the differences between the structure-based bioethics and the individual-based bioethics.

\section{Historical Lessons and Global Implications of Structure-Based Bioethics on Theory, Policy, Health System Reform Practices and Medical Professionalism}

The structure-based bioethics is not just suitable in the situation in the Chinese mainland. It has global significance under the circumstances of globalization, global health, and global health system reform.

Two lessons we can learn from the typical and extreme case in Tongren Hospital and the health system reform dilemma in the Chinese mainland are: First, health care system is, after all, welfare in nature, and second, the quality of medical professionalism depends on the quality of health care system (Liu Jitong, Guo Yan, Chen Ningshan et al., 2007). The health system reform in the Chinese mainland appears to be a project engaged in mutual adaptation with the social system, where doctors' behaviors are deeply affected by the social structure. The political restructuring and overall plan in the Chinese mainland, especially health system reform scheme, influence the moral philosophy and bioethics deeply. And the quality of bioethics depends on the quality of health system reform (Liu Jitong, 2010). The establishment of health system reform

Table 1.

Differences between the structure-based bioethics and the individual-based bioethics.

\begin{tabular}{ccc}
\hline Compared Items & Structure-based Bioethics & Individual-based Bioethics \\
\hline Nature & sociality or socialization & individuality or individualization \\
Origin & result of “social structure” & pure individualized life \\
Category & diversity and pluralism & singularity \\
Main manifestation & groups and occupations based & individual-based \\
Other influences & unique and high relation with periodical features and culture & less historical and cultural influences \\
\hline
\end{tabular}


scheme should take into consideration multiple factors, including doctor's sources of revenue and the financing model in hospitals. Payment for health care should not be directly made from the patients' pockets, but from the third party single-payer system.

The building of structure-based bioethics will contribute to optimizing the theory and policy of the health system reform, improving health system reform practices, and promoting medical professionalism.

Theoretically, the individual-based bioethics in the West is not transplantable to the Chinese mainland, where lots of bioethical misconducts of doctors should be explained by inappropriate social structure. The structure-based bioethics, however, emphasizes the interaction between morality and national policies and other social ethics, so as to understand the deciding power of macro social structure and moral philosophy structure that shapes personal behaviors and life, and to promote the transformation from condemning individuals to the establishment of systems (Liu Jitong, 2005). What this means to health system reform is a change from blaming the doctors to improving health system and systematic policies. It may also enrich the theoretical system of bioethics in Western society.

In this reform, the sociality of structure-based bioethics calls for a public understanding of bioethics, medical code of ethics, and medical professionalism. And such understanding is to be attained against a background of the social complexity in China (Liu Jitong, Yan Jun, Wang Mingxu et al., 2009). It also requires the understanding of bioethics, moral philosophy and the medical professionalism from the perspectives of social system and its structural function.

As for medical professionalism, the health industry, as part of social service, should follow the guidelines of the professionalism in the service. Therefore the value behind the medical services is the most important component of medical professionalism. We should abide by the Hippocratic Oath strictly, and regain patients' trust (Liu Jitong, Yan Jun, \& Kong Lingzhi, 2010). We should strengthen the humanities education for medical students to improve their medical professionalism. Besides, the introduction of social workers and social welfare system into the medical service area may also provide additional guarantee for medical professionalism and help meet the requirement of transform of biomedical model (Liu Jitong, 2006).

The structure-based bioethics follows the inner rules of sociality, which reminds us of two approaches in system building to solve the bioethical dilemma in the health system reform in the Chinese mainland and resolve the professionalism crisis of doctors. One is to regain the nature of "social welfare" in medical services; the other is to reinforce the building of the system of public welfare finance and health finance (Liu Jitong, 2008).

\section{Conclusion: Building Harmonious Doctor-Patient Relationship and Reconstructing Medical Professionalism}

The reform in health system in the Chinese mainland is facing an unprecedented structural dilemma, of which the structural dilemma of bioethics is erroneously considered as the main reason for the tension in the doctor-patient relationship and the decreasing of doctors' professionalism. It seems that the problem of "poor access and high fee" prevailing in the Chinese mainland would be gone if doctors were more ethically strong.
Therefore, the bioethics becomes the best perspective to observe the dilemma of the health system reform and doctor-patient relationship in the Chinese mainland.

Having discussed the health system reform in the Chinese mainland, compared the Western and Chinese bioethics, and identified the common goal of health to be the enhancement of the wellbeing of patients, the authors come up with a new theoretical model, namely structure-based bioethics. As a new concept created by the authors, it refers to the synthesis of opinions and principles to observe moral philosophy and code of ethics from the perspective of social system, social structure, and social interaction. Its opposition is individual-based bioethics. It is composed by such six main components as identifying its nature, main focus, research scope, research methods, and major function. In addition, the structure-based bioethics and the individual-based bioethics are compared from several perspectives so as to explain the sociality and diversity of the structure-based bioethics, and, with specific examples, illustrate its origins and main characteristics distinctive from the individual-based bioethics.

To sum up, the forgoing discussions on the policy implications of structure-based bioethics and, especially, the aspects of theoretical innovation, policy guidance, and medical professionalism have been conducted in hope of promoting the a change from condemning individuals to establishing systems. This requires a general understanding of the bioethics and medical professionalism against the background of social complexity, and suggests building social welfare system to achieve the common goals of healthcare. The final goal is to establish a new health system based on a systematic and structural consideration, to build a harmonious doctor-patient relationship, and to promote the medical humanities care and medical professionalism, so that the development of the reform in health system in the Chinese mainland can proceed in a systemized manner.

\section{REFERENCES}

China Hospital Management Association (2003). Yiwu renyuan bei ou bei hai qingkuang yanzhong yiwu renyuan quanli jixu baozhang 医 务人员被殴、被害情况严重，医务人员权利急需保障. Unpublished Raw Data.

China Youth Daily (2011). Tongren Yiyuan nü yisheng zao buming nanzi kan shang 同仁医院女医生遭不明男子砍伤. http://zqb.cyol.com/html/2011-09/16/nw.D110000zgqnb_20110916_ 4-07.htm

Engelhardt Jr., H. T. (1996). The foundation of bioethics. Oxford: Oxford University Press.

Fletcher, J. (1966). Situation ethics: The new morality. Philadelphia, PA: The Westminster Press.

Li Qi 李奇 (1994). Daode yu shehui shenghuo 道德与社会生活. Shanghai: Shanghai People's Publishing House.

Liu Jitong 刘继同 (2004a). Wei shenme weisheng zhengce hai buneng chengwei guoce 为什么卫生政策还不能成为“国策”? Zhongguo Weisheng, 7, 51-53.

Liu Jitong 刘继同 (2004b). Zuzhi xing geren zhuyi: Shehui zhuanxingqi zhongguo shehui de fuli wenhua yu lilun kuangjia 组织性个人 主义: 社会转型期中国社会的福利文化与理论框架. Zhongguo Shehui Kexue Pinglun, 2, 66-71

Liu Jitong 刘继同 (2005). Weisheng gaige kunjing chengyin de xitong jiegou fenxi yu hongguan zhanlue sikao 卫生改革困境成因的 系统结构分析与宏观战略思考. Zhongguo Weisheng Jingji, 24, 19-22. 
Liu Jitong 刘继同 (2006). Goujian hexie yihuan guanxi yu yiwu shehui gongzuo de zhuanye shiming 构建和谐医患关系与医务社会工 作的专业使命. Zhongguo Yiyuan Guanli, 26, 15-18.

Liu Jitong 刘继同, Guo Yan 郭岩, Chen Ningshan 陈宁珊 et al. (2007). Shenme shi hao de weisheng zhengce 什么是“好”的卫生政 策? Zhongguo Yiyuan Guanli, 27, 3-6.

Liu Jitong 刘继同 (2008). Weisheng caizheng xue gainian de hanyi, fanwei lingyu, jiben tezheng yu diwei zuoyong (shang) 卫生财政学 概念的涵义、范围领域、基本特征与地位作用(上). Zhongguo Weisheng Jingji, 27, 5-7

Liu Jitong 刘继同, Yan Jun 严俊, Wang Mingxu 王明旭 et al. (2009). Zhongguo yixue renwen, yixue zhiye jingshen de zhuyao yanjiu yiti yu zhidu hua jueding yinsu 中国医学人文、医学职业精神的主要研 究议题与制度化决定因素. Zhongguo Weisheng Zhengce Yanjiu, 10, 56-61.

Liu Jitong 刘继同 (2010). Lun yiyao weisheng tizhi gaige zhiliang 论 医药卫生体制改革质量. Weisheng Jingji Yanjiu, 11, 13-15.

Liu Jitong 刘继同, Yan Jun 严俊, \& Kong Lingzhi 孔灵芝 (2010). Zhongguo yixue renwen neihan jigou yu yiwu gongzuo zhidu jianshe 中国医学人文内涵结构与医务工作制度建设. Yixue Yu Shehui, 23, 11-13.

Macintyre, A. (1967). A short history of ethics. London: Routledge \& Kegan Paul plc.
Sidgwick, H. (1901). The methods of ethics. New York: The Macmillan Company.

Smart, J. J. C., \& Bernard, W. (1983). Utilitarianism: For and against. England: Cambridge University Press

Sun Muyi 孙慕义 (2007). Hou xiandai shengming shenxue-Shengming lunli xue de gainian tushi yu zhongjie bianlun 后现代生命神 学—生命伦理学的概念图式与终结辩论. Taipei: Wenfeng Cultural Enterprises Co. Ltd.

Sun Shuyin 孙树印 (2011). Xin xingshi xia yihuan jiufen chuli moshi tantao 新形势下医患纠纷处理模式探讨. Zhong Yiyao Guanli Zazhi, 19, 71-73.

Xu Zhiwei 许志伟 (2006). Zhongguo dangqian de yiliao weiji yu yihu renyuan de zhuanye zeren he shiming 中国当前的医疗危机与医护 人员的专业责任和使命. Yixue yu Zhexue (Renwen Shehui Yixue Ban), 27, 1-6.

Zhao Meijuan 赵美娟 (2005). Yixue renwen guanhuai ying guanhuai shenme he zenyang guanhuai-Meixue shijiao xia de renwen guaihuai jianshe 医学人文关怀应关怀什么和怎样关怀? 一一美学视角 下的人文关怀建设. Yixue yu Zhexue, 26, 26-28. 